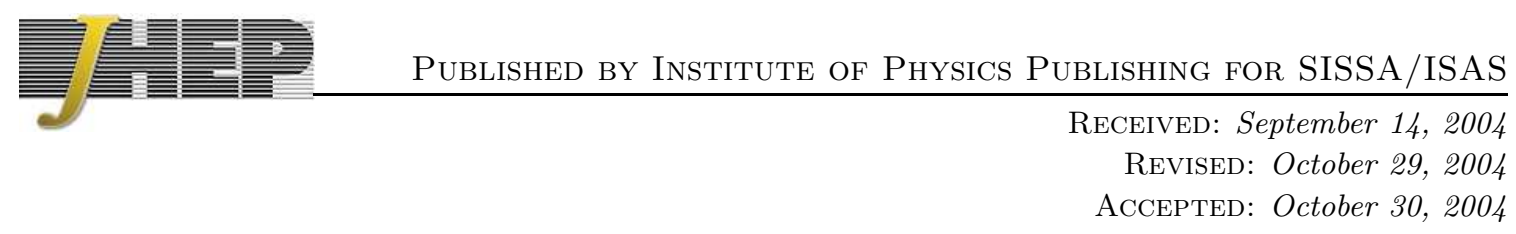

\title{
Phases of massive gravity
}

\section{Sergei L. Dubovsky}

Department of Physics, CERN Theory Division

CH-1211 Geneva 23, Switzerland, and

Institute for Nuclear Research of the Russian Academy of Sciences

60th October Anniversary Prospect, 7a, 117312 Moscow, Russia

E-mail: sergd@ms2.inr.ac.ru

ABSTRACT: We systematically study the most general Lorentz-violating graviton mass invariant under three-dimensional Eucledian group. We find that at general values of mass parameters the massive graviton has six propagating degrees of freedom, and some of them are ghosts or lead to rapid classical instabilities. However, there is a number of different regions in the mass parameter space where massive gravity is described by a consistent low-energy effective theory with cutoff $\sim \sqrt{m M_{P l}}$. This theory is free of rapid instabilities and vDVZ discontinuity. Each of these regions is characterized by certain finetuning relations between mass parameters, generalizing the Fierz-Pauli condition. In some cases the required fine-tunings are consequences of the existence of the subgroups of the diffeomorphism group that are left unbroken by the graviton mass. We found two new cases, when the resulting theories have a property of UV insensitivity, i.e. remain well behaved after inclusion of arbitrary higher dimension operators without assuming any fine-tunings among the coefficients of these operators, besides those enforced by the symmetries. These theories can be thought of as generalizations of the ghost condensate model with a smaller residual symmetry group. We briefly discuss what kind of cosmology can one expect in massive gravity and argue that the allowed values of the graviton mass may be quite large, affecting growth of primordial perturbations, structure formation and, perhaps, enhancing the backreaction of inhomogeneities on the expansion rate of the Universe.

KEywords: Spontaneous Symmetry Breaking, Space-Time Symmetries, Classical Theories of Gravity, Cosmology of Theories beyond the SM. 


\section{Contents}

1. Introduction 1

2. Stückelberg trick for Lorentz-violating massive gravity

3. Quadratic Goldstone action at the one-derivative level 8

3.1 Generic graviton masses: six propagating degrees of freedom and ghosts or classical instabilities 10

3.2 Phase $m_{0}^{2}=0$

3.3 Phase $m_{2}^{2}=m_{3}^{2}$

3.4 Phase $m_{2}^{2}=0$

3.5 Phase $m_{1}^{2}=0$

4. Higher-derivative terms, UV (in)sensitivity and unbroken gauge symmetries

5. Mixing with gravity and absence of the vDVZ discontinuity 22

6. Discussion 26

\section{Introduction}

During the last few years a significant progress in understanding of properties of massive gravity in four dimensions has been achieved. One of the first steps was the implementation of the Stückelberg trick in the Fierz-Pauli theory [1]. As a result, a clear unified picture of the apparently mysterious properties of the massive gravity has emerged. Namely, the explicitly covariant Stückelberg language made it clear that the famous van Dam-VeltmanZakharov (vDVZ) discontinuity [2, 3] and related non-linear effects [4, as well as the hidden sixth polarization mode of the massive graviton [0] are all consequences of the fact that the kinetic term in the Goldstone sector of the Fierz-Pauli theory is degenerate in the limit $M_{P l} \rightarrow \infty$. As a result, the theory is not well defined in the limit when gravity decouples and the cutoff energy of the Fierz-Pauli theory in the Minkowski background is extremely low,

$$
\Lambda_{3} \leq\left(M_{P l}^{2} m\right)^{1 / 3} \sim(1000 \mathrm{~km})^{-1},
$$

where $m$ is a graviton mass, which we take of order the current Hubble scale. It is worth noting that this may not necessarily imply that the Fierz-Pauli theory is unacceptable phenomenologicaly. Indeed, recently it was shown [6] (see also [7]) how similar problem [8, 9] present in the context of co-dimension one Dvali-Gabadadze-Porrati (DGP) model [10] may be cured by taking into account the effects of local curvature. It is unclear at the moment whether this mechanism can be implemented in the Fierz-Pauli case. 
The further progress in the four-dimensional theories of massive gravity is due to idea [11], that instead of Fierz-Pauli mass term, which completely breaks diffeomorphism invariance of the theory, one can introduce a graviton mass, breaking only the time reparametrization invariance,

$$
t \rightarrow t+\xi^{0}(t, x),
$$

while keeping the arbitrary time-dependent spatial diffeomorphisms

$$
x^{i} \rightarrow x^{i}+\xi^{i}\left(t, x^{i}\right) .
$$

The crucial difference with the Fierz-Pauli case is that in this model the Goldstone sector (consisting of a single scalar field) is well behaved in the limit when gravity is decoupled. At the linear level the resulting Lorentz non-invariant theory (dubbed "ghost condensate") is characterized by two parameters - the characteristic length and time scales. Interestingly, it appeares that both of these parameters can be substantially shorter than the inverse Hubble scale without contradicting to observations [12, 13]. ${ }^{1}$ It is worth mentioning, however, that in many respects ghost condensate is very different from what one would call a theory of massive graviton. In particular, in this model a massless transverse traceless mode is present in the spectrum.

Finally, very recently it was argued in ref. [15], that the generic Lorentz-violating graviton mass term, preserving euclidean symmetry of the three-dimensional space, ${ }^{2}$

$$
L_{m}=\frac{M_{P l}^{2}}{2}\left(m_{0}^{2} h_{00} h_{00}+2 m_{1}^{2} h_{0 i} h_{0 i}-m_{2}^{2} h_{i j} h_{i j}+m_{3}^{2} h_{i i} h_{j j}-2 m_{4}^{2} h_{00} h_{i i}\right),
$$

where $h_{\mu \nu}$ are perturbations about Minkowski metric, also leads to the theory with well behaved Goldstone sector in the decoupling limit. More precisely, it was shown to be true for $m_{0}=0$ and provided the other masses satisfy certain positivity conditions. As a result, this mass term does not lead neither to ghosts, nor to low strong coupling scale in the flat space. Unlike the ghost condensate model, the spectrum of the theory has a mass gap, and describes five propagating degrees of freedom. Still, the vDVZ discontinuity is absent in this model and the cutoff scale is given by

$$
\Lambda_{2} \sim \sqrt{m M_{P l}} \sim(0.1 \mathrm{~mm})^{-1} .
$$

However, one may be worried by the need to satify the fine-tuning relation

$$
m_{0}^{2}=0
$$

in this theory. This condition is remininscent of the Fierz-Pauli fine-tuning and may be a signal of the presence of the analogue of the Boulware-Deser mode and related instabilities.

This result indicates that the ghost condensate is unlikely to be a single consistent infrared modification of gravity and calls for the further systematic study of the Lorentzviolating graviton mass. This is the main task of the current paper.

\footnotetext{
${ }^{1}$ It was argued in ref. [14] that the limits can be much tighter due to efficient accretion of the ghost condensate by black holes. It is not clear at the moment, whether the steady state solutions found in ref. 14] are the physically relevant ones.

${ }^{2} \mathrm{~A}$ possibility to introduce mass terms of this type was also mentioned in ref. [16].
} 
We start in section 2 with the covariant formulation of the Lorentz-violating massive gravity. For this purpose we use a Stückelberg-like formalism. This formalism is a slightly more flexible version of one used in the Lorentz-invariant case [1]. As in the Lorentzinvariant case, graviton mass generically leads to the emergence of four new dynamical degrees of freedom - Golsdtone bosons corresponding to broken reparametrizations of four coordinates. An example of ghost condensate hints that the Goldstone sector can be made well behaved in the limit when gravity is decoupled by requiring that some residual subgroup of the diffeomorphism group is left. However, the group of diffeomorphisms has too many subgroups, so the case by case consideration of all possible patterns of symmetry breaking seems hopeless.

Instead, in section 3 we adopt the bottom-up approach and study the spectrum of the Lorentz-violating massive gravity at general values of masses $m_{i}$ in the decoupling limit $M_{P l} \rightarrow \infty$. In agreement with expectations of ref. [15] we find that at non-zero $m_{0}^{2}$ theory generically has either ghosts or exhibits rapid classical instabilities. However, we found a number of other fine-tuning relations ensuring the absence of pathologies in the Goldstone sector at the one-derivative level. Actually, the very possibility of the other choices was expected, as we know that region with $m_{0}^{2}>0$ and all other masses equal to zero correspond to the ghost condensate model with a well behaved Goldstone sector. What is interesting is that one obtaines the well behaved Goldstone sector under weaker assumptions as well.

In section 4 we discuss the effects of higher derivative terms. We argue, that, generically, one should assume an infinite number of fine-tunings in higher order terms to preserve the above attractive properties of the low-energy theory. It is worth stressing that this does not imply that these fine-tunings cannot be realized in some specific UV completions of massive gravity. This only means that a generic UV completion would result in a massive gravity theory with cutoff scale much lower than $\Lambda_{2}$.

However, we found two new cases when all the required fine-tunings can be ensured by the residual reparametrization symmetry. In both cases this symmetry is a subgroup of the residual symmetry present in the ghost condensate. Namely, in one case massive gravity is invariant under time independent spatial diffeomorphisms

$$
x^{i} \rightarrow x^{i}+\xi^{i}(x)
$$

and in other under time-dependent shifts of spatial coordinates

$$
x^{i} \rightarrow x^{i}+\xi^{i}(t)
$$

Both theories inherit from the ghost condensate the property of UV insensitivity. Namely, they remain well behaved under inclusion of all possible higher derivative operators suppressed by the cutoff $\Lambda_{2}$ and compatible with the residual reparametrization symmetry, without assuming any fine-tuning relations between their coefficients.

Some of other properties of these massive gravities are also similar to that of the ghost condensate. For instance, all three models have a scalar degree of freedom with peculiar dispersion relation

$$
\omega^{2} \propto p^{4}
$$


However, in many respects two new models are very different from the ghost condensate. For instance, the tensor graviton mode has non-zero mass in case of residual symmetry (1.4).

In section 5 we discuss effects due to mixing with gravity, and prove that vDVZ discontinuity is absent in the massive gravity models discussed here (both fine-tuned and UV insensitive).

We conclude in section 5 with a brief discussion of cosmology in massive gravity. We provide an example of a theory with massive tensor mode, where flat homogeneous cosmological solutions are identical to those in the Einstein theory. This implies that the graviton mass can be substantially larger than the current Hubble value. Consequently, graviton mass may modify in the interesting way the dynamics of primordial perturbations and structure formation or, even, gravitational dynamics at the (super)galactic scales. Even more intriguingly, there is a possibility, that the presence of small scale inhomogeneities may significantly affect the expansion rate in massive gravity, potentially providing a link between the onset of the cosmological acceleration and epoch of structure formation.

\section{Stückelberg trick for Lorentz-violating massive gravity}

In the most general sense by massive gravity we understand any theory described by the following action

$$
S=-M_{P l}^{2} \int d^{4} x \sqrt{-g} R+\int d^{4} x \sqrt{-g} F,
$$

where the first term is a usual Einstein-Hilbert term and $F$ is, generally speaking, an arbitrary function of metric components, their derivatives, and coordinates $t, x^{i}(i=1,2,3)$ itself. One can add some matter fields to this system, which we assume to be minimally coupled to the metric. In the current paper we will limit our consideration to the case when theory (2.1) admits empty Minkowski space-time as one of the solutions to the field equations. Also, following ref. [15], we assume that a low-energy effective theory for small perturbations around this solution is invariant under the Eucledian transformations of the three-dimensional space coordinates $x^{i}$. In particular, this implies that function $F$ has no explicit dependence on the coordinates. Furthemore, we assume that function $F$ depends on a single energy scale $\Lambda$.

Then, the quadratic theory near Minkowski background is described by the usual linearized Einstein-Hilbert action plus mass term of the form (1.2), where the overall mass scale $m$ is related to the parameters $\Lambda$ and $M_{P l}$ as

$$
\Lambda=\sqrt{m M_{P l}} .
$$

The key issue we address in the current paper is what conditions function $F$ should satisfy in order dynamics of metric perturbations near Minkowski background could be described by a low-energy effective field theory with cutoff $\Lambda$. It was shown in ref. 罒, that this is not possible in the Lorentz-invariant case. The convenient tool allowing to adress this issue is the Stückelberg formalism. Our version of this formalism is essentially equivalent to that used in ref. [1]. Let us nevertheless briefly describe it, both to make our presentation more self-contained and because we are using slightly more flexible version of the formalism to allow for Lorentz non-invariant situation. 
The first step is to introduce four scalar fields $\phi^{\mu}(\mu=0, \ldots, 3)$. These are Goldstone bosons corresponding to four broken diffeomorphisms

$$
x^{\mu} \rightarrow \xi^{\mu}(x)
$$

The dynamics of these fields is described by some sigma-model action. We assume that this action is characterized by a single energy scale $\Lambda$. The main requirement to this action is that when coupled to gravity it provides the following field configuration as a solution to the field equations

$$
\begin{aligned}
\phi^{\mu} & =\Lambda^{2} x^{\mu} \\
g_{\mu \nu} & =G_{\mu \nu},
\end{aligned}
$$

where $G_{\mu \nu}$ is some fixed metric (Minkowski metric in our case). Provided such a solution exists, one can write

$$
\begin{aligned}
g_{\mu \nu} & =G_{\mu \nu}+h_{\mu \nu} \\
\phi^{\mu} & =\Lambda^{2} x^{\mu}+\pi^{\mu}
\end{aligned}
$$

and study dynamics of small scalar and metric perturbations $\pi^{\mu}, h_{\mu \nu}$. This, by definition, will be a certain version of massive gravity in the space-time with metric $G_{\mu \nu}$. Indeed, given such a theory, one can fix the unitary gauge $\pi^{\mu}=0$ and recover the previous non-covariant formulation (2.1) of massive gravity. ${ }^{3}$

Inversely, given action (2.1) one always can make it explicitely covariant reintroducing the Goldstone fields $\phi^{\mu}$. Namely, everywhere in the action (2.1) one replaces metric $g_{\mu \nu}$ with its gauge transform

$$
g_{\mu \nu}(x) \rightarrow \hat{g}_{\mu \nu} \equiv \frac{\partial Y^{\lambda}(x)}{\partial x^{\mu}} \frac{\partial Y^{\rho}(x)}{\partial x^{\nu}} g_{\lambda \rho}(Y(x)) .
$$

Here $Y^{\mu}$ are component functions of the diffeomorphism

$$
\hat{Y}: x^{\mu} \rightarrow Y^{\mu}(x) .
$$

Analogously, in the presence of matter fields $\psi$ one replaces them everywhere with their images under gauge transformation. Clearly, fields $Y^{\mu}$ appear only in the non-covariant part of the action. In particular, no direct coupling between these fields and matter arises, provided the action for matter is covariant.

Then action (2.1) considered as a functional of the metric $g_{\mu \nu}$ and fields $Y^{\mu}$ is diffeomorphism invariant under conventional transformations of the metric, accompanied by the following transformations of the fields $Y^{\mu}$,

$$
Y^{\mu} \rightarrow\left(\xi^{-1} \circ \hat{Y}\right)^{\mu}
$$

\footnotetext{
${ }^{3}$ Note, that field equations following from the variations of the Goldstone fields are consequences of the other equations in the unitary gauge.
} 
where $\xi$ is a change of coordinates and $\circ$ is a natural multiplication of two diffeomorphisms. Transformation law (2.7) implies that component functions $Y^{\mu}$ do not transform as scalars under changes of coordinates. However, the transformation law for the component functions of the inverse mapping $\hat{Y}^{-1}$ is that of the scalar fields,

$$
\left(\hat{Y}^{-1}\right)^{\mu} \rightarrow\left(\hat{Y}^{-1} \circ \xi\right)^{\mu}
$$

These component functions are precisely the Goldstone fields $\phi^{\mu}$ introduced earlier,

$$
\phi^{\mu} \equiv\left(\hat{Y}^{-1}\right)^{\mu}
$$

Now, imagine that massive gravity in the unitary gauge (2.1) has a residual invariance under diffeomorphisms $\xi_{H}$ from a certain subgroup $\mathcal{H}$ of the whole group of diffeomorphisms. In covariant formalism this implies that the theory is invariant under the following transformations of component fields $Y^{\mu}$,

$$
Y^{\mu} \rightarrow\left(\hat{Y} \circ \xi_{H}\right)^{\mu}
$$

In terms of scalar Goldstone fields $\phi^{\mu}$ this implies that a group $\mathcal{G}$ of internal global symmetries of the Goldstone action contains group $\mathcal{H}$ as its subgroup, i.e. transformations of the type

$$
\phi^{\mu} \rightarrow \xi_{H}^{\mu}(\phi)
$$

are symmetries of the Goldstone action. One could think that it is impossible to construct non-trivial actions with such symmetries for large enough groups $\mathcal{H}$. However, the Stückelberg trick described above allows to construct a lot of such actions starting from the massive gravity in the unitary gauge.

If, in addition, a group of internal symmetries $\mathcal{G}$ contains the common subgroup with the group of isometries of the metric $G_{\mu \nu}$, then vacuum (2.2), (2.3) is invariant under the diagonal combination of these two subgroups.

To finish this general discussion the following comment is in order. One may question the assumption that the massive gravity contains just metric and Goldstone bosons. Indeed, in the case of gauge fields Higgs mechanism implies the presence of at least one additional scalar field - the radial mode of the Higgs field. Presumably, the successful implementation of the Higgs mechanism in gravity (i.e., a theory of massive graviton with UV cutoff at the Planck scale) would also require some extra fields. Unfortunately, such a theory is not constructed yet. ${ }^{4}$ In this situation, the best we can do is to introduce the minimal set of fields which is required by the consistency. Then there is nothing wrong in solving the resulting non-linear equations, provided one restricts oneself to the classical solutions tractable within the low-energy effective theory. Tractable means here, that the theory of small perturbations around the solution has no pathologies leading to rapid instabilities and has large enough cutoff scale. Solutions that do not satisfy these criteria cannot be studied without more detailed knowledge of the UV completed theory.

\footnotetext{
${ }^{4}$ See, e.g., ref. 17 for a recent attempt in this direction, making use of the vector field.
} 
Let us illustrate the above general discussion by some concrete examples. For instance, the Fierz-Pauli theory corresponds to the choice of the Minkowski metric as a background metric $G_{\mu \nu}$, and the four-dimensional Poincare group (i.e., the whole group of isometries of the Minkowski space-time) as a group ${ }^{5} \mathcal{G}$.

Ghost condensate corresponds to the degenerate situation when action does not depend on the fields $\phi^{i}$ at all. Formally, one can say that in this case a group of internal symmetries $\mathcal{G}$ is a subgroup of the diffeomorphism group shifting time by a constant.

The Lorentz-violating massive gravity considered in ref. [15] is also a theory of massive gravity in the Minkowski space-time. It corresponds to the choice of the subgroup of the $4 \mathrm{~d}$ Poincare group generated by space-time shifts and rotations of space coordinates as a group of internal symmetries. Then the most general Goldstone action has the following form at the one-derivative level

$$
\int d^{4} x \Lambda^{4} \sqrt{-g} F\left(X, Y^{i j}, V^{i}, Z\right)
$$

where $X, Y^{i j}, V^{i}$ and $Z$ are the following scalar quantities

$$
\begin{aligned}
X & =\frac{g^{\mu \nu} \partial_{\mu} \phi^{0} \partial_{\nu} \phi^{0}}{\Lambda^{4}} \\
Y^{i j} & =\frac{g^{\mu \nu} \partial_{\mu} \phi^{i} \partial_{\nu} \phi^{j}}{\Lambda^{4}} \\
V^{i} & =\frac{g^{\mu \nu} \partial_{\mu} \phi^{0} \partial_{\nu} \phi^{i}}{\Lambda^{4}} \\
Z & =\frac{\epsilon^{\mu \nu \lambda \rho} \partial_{\mu} \phi^{0} \partial_{\nu} \phi^{1} \partial_{\lambda} \phi^{2} \partial_{\rho} \phi^{3}}{\left(\sqrt{-g} \Lambda^{8}\right)} .
\end{aligned}
$$

In the rest of the paper by massive gravity (or Lorentz-violating massive gravity) we imply this particular model. Note that $Z^{2}$ can be written as a function of $X, Y^{i j}, V^{i}$, so that different functions $F$ in eq. (2.10) may lead to the same Goldstone action. The Latin indices $i, j=1,2,3$ are converted using the $3 \mathrm{~d}$ Kronecker symbol $\delta_{i j}$ in eq. (2.10). The energy scale $\Lambda$ is a naive UV cutoff of this model which is assumed to be much lower than $M_{P l}$. As we explain later, in certain circumstances the actual UV cutoff may be significantly lower.

Clearly, for constant metrics $g_{\mu \nu}$ the field configuration (2.2) is always a solution to the field equations following from the action (2.10). To satisfy the second condition (2.3) for a Minkowski space-time one should find two positive constants $a, b>0$ such that the energy-momentum tensor of the sigma-model (2.10) is zero in the background metric

$$
\eta_{\mu \nu}=\operatorname{diag}(a,-b,-b,-b) \text {. }
$$

This condition translates into a following pair of equations

$$
2 F_{X} X=\frac{2}{3} F_{Y^{i j}} Y^{i j}=F-Z F_{Z}
$$

\footnotetext{
${ }^{5}$ Strictly speaking, in this way one obtains some general Lorentz-invariant theory of massive graviton. To ensure that the graviton mass has the Fierz-Pauli form, the sigma-model action should satisfy additional fine-tuning conditions.
} 
for the values of function $F$ and its first derivatives

$$
F_{X} \equiv \frac{\partial F}{\partial X}, \quad F_{Y^{i j}} \equiv \frac{\partial F}{\partial Y^{i j}}, \quad F_{Z} \equiv \frac{\partial F}{\partial Z}
$$

at the point

$$
X=a^{-1}, \quad Y^{i j}=-b^{-1} \delta^{i j}, \quad V^{i}=0, \quad Z=a^{-1 / 2} b^{-3 / 2} .
$$

It is straightforward to check that for a generic function $F$ one can always find $a$ and $b$ such that these conditions are met. Then masses $m_{0}^{2}, \ldots, m_{4}^{2}$ are combinations of the first and second derivatives of the function $F$ at this point. The overall mass scale $m$ is related to $\Lambda$ as

$$
\Lambda \sim \sqrt{M_{P l} m} .
$$

\section{Quadratic Goldstone action at the one-derivative level}

Let us first study the Goldsone sector for general values of masses $m_{i}^{2}$ in the absence of gravity. To obtain the quadratic Goldstone lagrangian one plugs the "pure gauge" metric perturbation

$$
h_{\mu \nu}=\partial_{\mu} \pi_{\nu}+\partial_{\nu} \pi_{\mu}
$$

in the action (1.2). The result is [15]

$$
\begin{aligned}
L=M_{P l}^{2}( & 2 m_{0}^{2}\left(\partial_{0} \pi_{0}\right)^{2}+m_{1}^{2}\left(\partial_{0} \pi_{i}\right)^{2}+m_{1}^{2}\left(\partial_{i} \pi_{0}\right)^{2}+\left(4 m_{4}^{2}-2 m_{1}^{2}\right) \pi_{0} \partial_{0} \partial_{i} \pi_{i}- \\
- & \left.m_{2}^{2}\left(\partial_{i} \pi_{j}\right)^{2}-\left(m_{2}^{2}-2 m_{3}^{2}\right)\left(\partial_{i} \pi_{i}\right)^{2}\right) .
\end{aligned}
$$

This lagrangian can be partially diagonalised by decomposing fields into components corresponding to different irreducible representations of the three-dimensional Eucledian symmetry group. The vector sector contains transverse part $\pi_{i}^{T}$ of the fields $\pi^{i}$,

$$
\partial^{i} \pi_{i}^{T}=0 .
$$

The high-energy action in this sector depends only on masses $m_{1}^{2}$ and $m_{2}^{2}$ and has the following form

$$
L=M_{P l}^{2}\left(m_{1}^{2}\left(\partial_{0} \pi_{i}^{T}\right)^{2}-m_{2}^{2}\left(\partial_{i} \pi_{j}^{T}\right)^{2}\right) .
$$

In general, this action is well behaved for

$$
m_{1}^{2}, m_{2}^{2}>0
$$

Indeed, if $m_{1}^{2}$ is negative, then $\pi_{i}^{T}$ become ghosts, while if $m_{1}^{2}$ is positive and $m_{2}^{2}$ is negative then there are classical instabilities at all spatial momenta. Points where at least one of these masses is zero are special. Thus conditions

$$
\begin{aligned}
& m_{1}^{2}=0 \\
& m_{2}^{2}=0
\end{aligned}
$$


are candidates on the role of fine-tuning relations. At the moment we proceed under assumption that condition (3.3) is satisfied and discuss later what happens if one or both of eqs. (3.4), (3.5) hold. It is worth recalling [15] also, that $m_{2}^{2}$ is a mass of the transversetraceless tensor graviton mode.

The scalar perturbations are $\pi_{0}$ and $\pi_{L}$, where the latter is defined as

$$
\pi_{i}=\frac{1}{\sqrt{-\partial_{i}^{2}}} \partial_{i} \pi_{L} .
$$

Their lagrangian has the following form

$$
\begin{aligned}
L=M_{P l}^{2} & \left(2 m_{0}^{2}\left(\partial_{0} \pi_{0}\right)^{2}+m_{1}^{2}\left(\partial_{0} \pi_{L}\right)^{2}+m_{1}^{2}\left(\partial_{i} \pi_{0}\right)^{2}-\right. \\
& \left.-\left(4 m_{4}^{2}-2 m_{1}^{2}\right) \pi_{0} \partial_{0} \sqrt{-\partial_{i}^{2}} \pi_{L}-2\left(m_{2}^{2}-m_{3}^{2}\right)\left(\partial_{i} \pi_{L}\right)^{2}\right) .
\end{aligned}
$$

In the Fourier space the field equations following from this lagrangian are

$$
M\left(\begin{array}{l}
\pi_{0} \\
\pi_{L}
\end{array}\right)=0,
$$

where $M$ is the following $2 \times 2$ matrix,

$$
M=\left(\begin{array}{cc}
\alpha \nu^{2}+1 & -i \mu \nu \\
i \mu \nu & \nu^{2}-\beta
\end{array}\right),
$$

where

$$
\begin{aligned}
\nu & =\frac{\omega}{p}, \\
\alpha & =\frac{2 m_{0}^{2}}{m_{1}^{2}}, \quad \mu=\frac{2 m_{4}^{2}-m_{1}^{2}}{m_{1}^{2}}, \quad \beta=2 \frac{m_{2}^{2}-m_{3}^{2}}{m_{1}^{2}},
\end{aligned}
$$

with $\omega$ and $p$ being energy and absolute value of the three-momentum correspondingly. Generically, there are two propagating degrees of freedom in the scalar sector. Their dispersion relations are determined by the condition that the determinant $d$ of the matrix $M$ is zero,

$$
d \equiv \alpha \nu^{4}+\nu^{2}\left(1-\beta \alpha-\mu^{2}\right)-\beta=0 .
$$

There are some special values of the parameters, where the number of degrees of freedom in the scalar sector is reduced. It may happen either when one of the roots of eq. (3.9) goes to infinity, so that the dispersion relation for the corresponding mode takes form

$$
p^{2}=0
$$

or when one of the roots goes to zero, so that the corresponding dispersion relation is

$$
\omega^{2}=0 \text {. }
$$

The first option is realized for

$$
\alpha=0
$$


so that the order of the equation (3.9) reduces by one. This is the case considered in ref. [15]. There is a single propagating mode in the scalar sector in this case. Actually, there is a possibility to reduce the order of the on-shell equation even further. This happens when

$$
\alpha=0, \quad \mu^{2}=1 .
$$

In this case there are no propagating degrees of freedom in the scalar sector. The second option (3.11) is realized for

$$
\beta=0
$$

Both scalar degrees of freedom have zero energy and do not propagate if

$$
\beta=0, \quad \mu^{2}=1
$$

Finally, it may happen that one of the fine-tuning relations (3.12), (3.13) and one of (3.14), (3.15) are satisfied simultaniously. For instance, the Fierz-Pauli fine-tuning implies that both conditions (3.13) and (3.15) hold (with $\mu=1$ ).

Actually, we are making things somewhat too simple saying that the above special cases correspond to the reduction in the number of degrees of freedom. This is literally the case at the quadratic level in the limit when gravity is decoupled and in the absence of higher derivative terms in the Goldstone sector. However, one may be worried that inclusion of any of these effects strongly modifies the whole picture. This really happens in some cases, and more detailed discussion of these effects will be presented in sections 1 and 5 The oversimplified picture is enough, however, for the main purpose of this section - to identify regions in the mass space, where obvious pathologies are absent in the low-energy effective theory. Let us now perform case by case analysis of different possibilities.

\subsection{Generic graviton masses: six propagating degrees of freedom and ghosts or classical instabilities}

To start with, let us consider the most general case when none of the above fine-tuning relations holds, so that there are two propagating degrees of freedom in the scalar sector, and massive graviton has six polarization modes in total. Let us check whether it is possible then to chose parameters $\alpha, \mu, \beta$ in such a way that there are no ghosts or classical instabilities.

Formally the problem is formulated as follows. At any given values of the parameters $\alpha, \mu, \beta$ and positive $\nu^{2}$ the hermitean matrix $M$ has two real eigenvalues $\lambda_{ \pm}\left(\nu^{2}\right)$ given by

$$
\lambda_{ \pm}=\frac{t \pm \sqrt{t^{2}-4 d}}{2}
$$

where $t$ and $d$ are trace and determinant of the matrix $M$. Also there are two values $\nu_{1,2}^{2}$ where the on-shell condition (3.9) holds, i.e. one of the eigenvalues is zero at this point.

The question is whether it is possible to find values of the parameters such that the following two conditions are met, 
- Absence of the classical instability,

$$
\nu_{1,2}^{2}>0
$$

- Absence of ghosts

$$
\left.\frac{\partial \lambda_{ \pm}\left(\nu^{2}\right)}{\partial \nu^{2}}\right|_{\nu^{2}=\nu_{1,2}^{2}}>0 .
$$

Let us prove that this is impossible. The first observation is that to avoid ghosts it is necessary that each of the eigenvalues $\lambda_{ \pm}(\nu)$ has one zero. Indeed, if one of them has two zeros and another none than the derivative in one of the zeros is necessarily negative. Similar situation happens in a theory of a single scalar field with four derivative kinetic term.

Now, note that eq. (3.9) implies that one of the conditions for the absence of the classical instability is

$$
\frac{\beta}{\alpha}<0
$$

Let us first discuss the case $\alpha<0, \beta>0$. Here, in the limit of large $\nu^{2}$ the determinant of the matrix $M$ is

$$
d=\alpha \nu^{4}+\mathcal{O}\left(\nu^{2}\right)<0 .
$$

Consequently, the smaller eigenvalue $\lambda_{-}$is negative at large $\nu^{2}$ in this case. This implies, that derivative of $\lambda_{-}$in its zero is negative, and this zero corresponds to the ghost.

In the another case $\alpha>0, \beta<0$ the trace of the matrix $M$,

$$
t=(\alpha+1) \nu^{2}+1-\beta
$$

is positive definite. This implies, that $\lambda_{+}$is also positive definite. Consequently, $\lambda_{-}$has two zeroes, and one of them corresponds to the ghost.

\subsection{Phase $m_{0}^{2}=0$}

Let us now consider the case when the only fine-tuning relation which holds is (3.12). This is the case considered in ref. 15.

For $\alpha=0$ one of the solutions $\nu^{2}$ to the on-shell condition (3.9) becomes infinite. The only remaining solution is

$$
\nu_{0}^{2}=\frac{\beta}{1-\mu^{2}}
$$

Let us first consider the case

$$
\mu^{2}>1
$$

Then positiveness of $\nu_{0}^{2}$ implies that

$$
\beta<0 .
$$

As before, in this case the trace (3.20) of the matrix $M$ is positive for all positive $\nu^{2}$, so $\lambda_{+}$is also positive and $\nu_{0}^{2}$ is a zero of $\lambda_{-}$. On the other hand the determinant $d$ of matrix $M$ is now equal to

$$
d=\nu^{2}\left(1-\mu^{2}\right)-\beta
$$


and negative at large $\nu^{2}$. Consequently, $\lambda_{-}$is negative at large $\nu^{2}$ and has a negative derivative in its zero $\nu_{0}^{2}$. So the only dynamical degree of freedom in the scalar sector is a ghost here.

Let us now consider the opposite case

$$
\mu^{2}<1
$$

Then in order to avoid classical instabilities $\beta$ should be positive,

$$
\beta>0 .
$$

In this case both determinant (3.22) and trace (3.20) are positive at large $\nu^{2}$. Consequently, both eigenvalues $\lambda_{ \pm}$are positive there as well. This implies, that $\nu_{0}^{2}$ is a zero of the smaller eigenvalue $\lambda_{-}$, and that derivative of this eigenvalue in its zero is positive.

Furthemore, if in addition to eq. (3.12) one also has $\mu^{2}=1$, then the second root of the dispersion relation (3.9) goes to infinity. The determinant of matrix $M$ is equal to $-\beta$ and non-zero in this case, so one can use the inverse of this matrix as a propagator in the perturbation theory (for $\beta \neq 0$ ). The propagating scalar degrees of freedom and obvious pathologies are absent in the Goldstone sector in this case. If $\beta$ is equal to zero, then both fine-tuning relations (3.13) and (3.15) hold and we are in a situation generalizing that in the Fierz-Pauli theory. Here the quadratic part of the Goldstone sector is zero, so one cannot organize perturbation theory in this sector in the limit when gravity is decoupled.

Finally, there is a possibility that fine-tuning conditions (3.12) and (3.14) are satisfied simultaneously. Here the second mode in the scalar sector has dispersion relation of the form (3.11) and is not propagating as well. There are no obvious pathologies in the Goldstone sector in this case, provided $\mu^{2} \neq 1$.

To summarize, Goldstone sector is free of obvious pathologies in the high-energy limit at $m_{0}^{2}=0$, provided the graviton masses satisfy the following conditions

$$
m_{0}^{2}=0, \quad m_{1}^{2}, m_{2}^{2}>0, \quad m_{1}^{2}>m_{4}^{2}>0, \quad m_{2}^{2}>m_{3}^{2}
$$

or

$$
m_{0}^{2}=0, \quad m_{1}^{2}, m_{2}^{2}>0, \quad\left(m_{1}^{2}-m_{4}^{2}\right) m_{4}^{2}=0, \quad m_{2}^{2} \neq m_{3}^{2}
$$

or

$$
m_{0}^{2}=0, \quad m_{1}^{2}, m_{2}^{2}>0, \quad m_{2}^{2}=m_{3}^{2}, \quad\left(m_{1}^{2}-m_{4}^{2}\right) m_{4}^{2} \neq 0 .
$$

In the bulk of this phase one of the modes in the scalar sector has dispersion relation (3.10) and is not dynamical, so in total there are three propagating degrees of freedom in the Goldstone sector.

We will discuss later what happens when either $m_{1}^{2}$ or $m_{2}^{2}$ is zero. Already now it is clear that if in any of the above situations one has

$$
m_{2}^{2}=0
$$

then nothing changes in the scalar sector, while both vector degrees of freedom become non-dynamical and tensor mode is massless. 
The above conditions are in agreement with those found in ref. [15]. The difference is that we also studied here what happens on the boundaries of the region found in that work. Another difference is that an extra condition $4 m_{2}^{2}>m_{4}^{2}$ was imposed there. We will discuss the origin of this extra condition and whether it is really necessary in section .

\subsection{Phase $m_{2}^{2}=m_{3}^{2}$}

In this subsection we proceed under assumption that both conditions (3.3) are satisfied, and study what happens if $m_{0}^{2}$ is non-zero and fine-tuning relation (3.14 holds. Determinant $d$ of the matrix $M$ takes the following form in this case

$$
d=\nu^{2}\left(\alpha \nu^{2}+1-\mu^{2}\right)
$$

while its trace $t$ is

$$
t=(\alpha+1) \nu^{2}+1
$$

One of the solutions of the on-shell equation (3.9) is $\nu^{2}=0$ and does not propagate. Note, that this mode always corresponds to a zero of the smaller eigenvalue $\lambda_{-}$, because trace (3.27) is positive at $\nu^{2}=0$. The second solution of the on-shell equation is

$$
\nu_{23}^{2}=\frac{\mu^{2}-1}{\alpha} .
$$

Now, for $\alpha<0$ the determinant (3.26) is negative at large $\nu^{2}$, and, consequently, $\lambda_{-}$is also negative there, while $\lambda_{+}$is positive. The only possibility to have $\nu_{23}^{2}$ positive in this case is if $\nu_{23}^{2}$ is also a zero of $\lambda_{-}$, and if the derivative of $\lambda_{-}$is negative at $\nu_{23}^{2}$. Hence, one inevitably has classical instability or ghost for $m_{2}^{2}=m_{3}^{3}$ and $m_{0}^{2}<0$ (assuming conditions (3.3) are satisfied).

For $\alpha>0$ both trace (3.27) and determinant (3.26) are positive at large $\nu^{2}$, and consequently $\lambda_{+}$and $\lambda_{-}$are also both positive there. Therefore, provided $\nu_{23}^{2}$ is positive, it is a zero of $\lambda_{-}$in this case, the sign of the corresponding derivative is positive and there are no obvious pathologies in the Goldstone sector.

We have already discussed what happens if $\alpha=0$. Another possibility is that $\mu^{2}=1$, i.e. fine-tuning relation (3.15) is realized. In this case, there are also no obvious pathologies in the Goldstone sector. To summarize, there is a phase

$$
m_{2}^{2}=m_{3}^{2}>0, \quad m_{1}^{2}>0, \quad m_{0}^{2}>0, \quad m_{4}^{2}>m_{1}^{2} \quad \text { or } \quad m_{4}^{2}<0
$$

or

$$
m_{2}^{2}=m_{3}^{2}>0, \quad m_{1}^{2}>0, \quad\left(m_{4}^{2}-m_{1}^{2}\right) m_{4}^{2}=0, \quad m_{0}^{2} \neq 0,
$$

where Goldstone sector is well defined in the limit when gravity decouples. In the bulk of this phase one of the modes in the scalar sector has dispersion relation (3.11) and is not dynamical, so in total there are three propagating degrees of freedom in the Goldstone sector.

As above, nothing changes in the scalar sector if one takes in additon $m_{2}^{2}=0$ so that there are no propagating degrees of freedom in the vector sector and tensor mode is massless. 


\subsection{Phase $m_{2}^{2}=0$}

Let us discuss now what happens when mass $m_{2}^{2}=0$, so that gradient terms for the vector modes vanishes and tensor modes are massless. The above discussion has already covered this case for positive $m_{1}^{2}$. If, in addition to $m_{2}^{2}, m_{1}^{2}$ is also zero, then the whole quadratic lagrangian in the vector sector is zero in the limit when gravity decouples, so the Goldstone sector is not well behaved in this limit (the only exception is the ghost condensate case, when there are unbroken time-dependent spatial diffeomorphisms, so that Goldstones $\pi^{i}$ do not show up at all). So the only remaining possibility is that $m_{1}^{2}$ is negative,

$$
m_{1}^{2}<0
$$

This is possible because vector modes have zero energy and do not propagate for $m_{2}^{2}=0$.

The analysis of the scalar sector proceeds now very similar to what has been done above. The only difference is that the condition that there are no ghosts in the scalar sector is now opposite to (3.18),

$$
\left.\frac{\partial \lambda_{ \pm}\left(\nu^{2}\right)}{\partial \nu^{2}}\right|_{\nu^{2}=\nu_{1,2}^{2}}<0 .
$$

We will skip technical details and present just the results. As above, there are pathologies in the scalar sector for generic values of the parameters $\alpha, \mu, \beta$. For $\alpha=0$ the allowed region is

$$
m_{0}^{2}=m_{2}^{2}=0, \quad m_{1}^{2}<0, \quad m_{3}^{2}<0, \quad m_{4}^{2}<m_{1}^{2} \quad \text { or } \quad m_{4}^{2}>0
$$

or

$$
m_{0}^{2}=m_{2}^{2}=0, \quad m_{1}^{2}<0, \quad m_{3}^{2}=0, \quad m_{4}^{2}\left(m_{4}^{2}-m_{1}^{2}\right) \neq 0
$$

or

$$
m_{0}^{2}=m_{2}^{2}=0, \quad m_{1}^{2}<0, \quad m_{4}^{2}\left(m_{4}^{2}-m_{1}^{2}\right)=0, \quad m_{3}^{2} \neq 0 .
$$

For $\beta=0$ one has

$$
m_{2}^{2}=m_{3}^{2}=0, \quad m_{1}^{2}<0, \quad m_{0}^{2}>0, \quad m_{1}^{2}<m_{4}^{2}<0
$$

or

$$
m_{2}^{2}=m_{3}^{2}=0, \quad m_{1}^{2}<0, \quad m_{0}^{2}=0, \quad m_{4}^{2}\left(m_{4}^{2}-m_{1}^{2}\right) \neq 0
$$

or

$$
m_{2}^{2}=m_{3}^{2}=0, \quad m_{1}^{2}<0, \quad m_{4}^{2}\left(m_{4}^{2}-m_{1}^{2}\right)=0, \quad m_{0}^{2} \neq 0 .
$$

We see, that unlike in the previous cases, fine-tuning $m_{2}^{2}=0$ alone is never enough to make Goldstone sector free of pathologies. So, probably, there is no need to pick out the case $m_{2}^{2}=0$ into a separate phase. 
3.5 Phase $m_{1}^{2}=0$

The last remaining option is that

$$
m_{1}^{2}=0,
$$

so that vector modes have dispersion relation of the form (3.10) and do not propagate. This case was mentioned in ref. [15] as a potentially interesting. In this case the field equation in the scalar sector still has the form (3.7) with matrix $M$ being equal to

$$
M=\left(\begin{array}{lc}
2 m_{0}^{2} \nu^{2} & -2 i m_{4}^{2} \nu \\
2 i m_{4}^{2} \nu & 2\left(m_{3}^{2}-m_{2}^{2}\right)
\end{array}\right) .
$$

Therefore, the on-shell condition in this case takes the following form,

$$
d=\left(4 m_{0}^{2}\left(m_{3}^{2}-m_{2}^{2}\right)-4 m_{4}^{4}\right) \nu^{2}=0 .
$$

If

$$
m_{0}^{2}\left(m_{3}^{2}-m_{2}^{2}\right)-m_{4}^{4}=0,
$$

then matrix $M$ is degenerate, so that one cannot use its inverse as a propagator in the perturbation theory and Goldstone sector is not well behaved in the decoupling limit. However, for any other values of parameters Goldstone sector is well behaved, and there are no propagating vector and scalar modes in the theory (while tensor mode is massive).

\section{Higher-derivative terms, UV (in)sensitivity and unbroken gauge sym- metries}

In this section we continue the study of the Goldstone sector in the limit when gravity is decoupled and discuss how the effects of higher derivative terms affect the conclusions of section 3. Let us, however, first discuss what happens when the fine-tuning conditions discussed above are slightly violated. Indeed, if these fine-tuning condtions are not ensured by symmetries, then they are not stable at the quantum level, so one should check that the low-energy effective theory remains well behaved if one allows for small violations of fine-tuning relations. The result of such violation is that the non-propagating degrees of freedom become dynamical. Then, there are two different situations depending on whether the non-propagating degree of freedom had dispersion relation of the form (3.10) or (3.11).

Let us first discuss what happens upon small violation of the dispersion relation (3.11). In the vicinity of the point where this dispersion relation holds, the corresponding degree of freedom has dispersion relation of the following form

$$
\omega^{2}=\epsilon p^{2},
$$

where $\epsilon$ is a small parameter determined by the distance to the fine-tuned point in the mass space. Recall, that the analysis of subsection 3.1 implies that in massive gravity either $\epsilon<0$ leading to classical instability at all momenta, or $\epsilon>0$ and the corresponding 
degree of freedom is a ghost. ${ }^{6}$ However, in both cases these instabilities do no present a problem from the viewpoint of the low-energy effective field theory, provided $\epsilon$ is small enough. Indeed, for negative $\epsilon$ the rate $\Gamma$ for the development of classical instability is smaller than

$$
\Gamma \sim \omega \lesssim|\epsilon|^{1 / 2} \Lambda
$$

and can be made arbitrarily small by chosing a small enough $|\epsilon|$, while keeping the cutoff value $\Lambda$ constant.

If $\epsilon$ is positive, then the corresponding degree of freedom is a ghost, and there is a quantum instability related to the possibility to produce it from the vacuum together with normal particles of positive energy. However, at small $\epsilon$ the maximum (negative) energy of the ghost quanta available in the regime of validity of the low-energy effective field theory is very small so that the rate of development of quantum instability is strongly suppressed by the phase volume.

Definitely, in both cases there is an instability in the low-energy effective theory, so rigorously speaking the latter is not well defined. However, this is not important for any practical purposes provided the time-scale for the development of instability is long enough. In other words, in all practical situations there is an IR cutoff at long time scales (at least at the age of the Universe).

One could be confused by the fact, that we allow to have ghosts in the low-energy effective theory. Recall, (see, e.g., ref. [18, 19] for a recent discussion) that ghosts imply quantum instability of the vacuum due to the possibility of their creation accompanied by normal particles of positive energy. In Lorentz-invariant theory the rate of the development of this instability diverges due to the infinite phase volume, unless one introduces Lorentzviolating cutoff. Then the rate is proportional to the value of the cutoff. In particular, this implies that if the only dimensionfull parameter in the theory is the cutoff $\Lambda$ itself (as is the case in the Goldstone sector, where perturbativity requires that the actual cutoff should be just slightly lower than $\Lambda$ ), then the rate of instability is also of order $\Lambda$. One way to make this situation tractable is to introduce a cutoff at the significantly lower scale.

However, in the Lorentz non-invariant situation it may happen that the dispersion relation is such, that the highest (negative) available energy of the ghost quanta is very small (see, e.g. dispersion relation (5.18) which we discuss later). It is even possible, that the energy has a maximum at a certain value of momentum and goes to zero at higher momenta. In this case processes with substantial production of energy involve a large number of ghosts and can be very strongly suppressed even at relatively large values of the coupling constants. It may be even possible that there is no need for the explicit Lorentz-violating cutoff to make the rate of instability finite - Lorentz-violating dynamics of the system itself may play a role of such a cutoff. A somewhat degenerate example of a situation of this type happens in quantum cosmology, where scale factor of the Universe is a ghost with zero energy.

\footnotetext{
${ }^{6}$ Actually, our analysis does not exclude also a situation, when $\epsilon$ is negative and the corresponding degree of freedom is a ghost, i.e. both types of instabilities are present simultaneously.
} 
We believe that these issues deserve further studies. In any case, it is clear that if the maximum frequency of the ghost quanta is smaller than the inverse age of the Universe, then time uncertainty principle makes it nonsensible to speak about production of ghosts.

Let us now discuss what happens when the dispersion relation of the form (3.10) is slightly violated. In this case one has a mode with dispersion relation

$$
\epsilon \omega^{2}=p^{2}
$$

which is, in our case, again either indicates the presence of classical instability at $\epsilon<0$ or a ghost at $\epsilon>0$. Here the low values of momenta,

$$
p \lesssim|\epsilon|^{1 / 2} \Lambda
$$

are dangerous. Indeed, at higher momenta the presence or absence of instability depends on the structure of the theory above the cutoff energy, i.e. out of the domain of applicability of the low-energy effective field theory, so one can consistently assume that instability is absent. To eliminate the dangerously rapid instability at the small values of the momentum one needs some IR regulator. This regulator can be provided, for instance, by the finite size of the system, or by the Hubble expansion (in some similarity to how Jeans instability is eliminated at large length scales). Another option (which, as we discuss in section 5 , is realized, e.g., for $m_{0}^{2}=0$ phase) is that mixing with gravity leads to the following modification of the dispersion relation (4.2),

$$
\epsilon \omega^{2}=p^{2}+m^{2}
$$

where $m^{2}$ should be positive.

To summarize, there is an interesting complementarity of two situations (4.1) and (4.2). Namely, in the first case IR cutoff in time and UV cutoff in space are needed to stabilize the theory, while in the second case one needs IR cutoff in space and UV cutoff in time. ${ }^{7}$

Having discussed what happens upon small departure from the dispersion relations (3.10) and (3.11) at the one-derivative level, let us turn to the effects of the higher derivative terms. Generically, they will also make the would be non-dynamical degrees of freedom dynamical. For instance, generic higher derivative terms would lead to the following modification of the dispersion relation (3.11)

$$
\omega^{2}=a \frac{p^{4}}{\Lambda^{2}}+\cdots,
$$

where $a$ is a coefficient of order one. This is precisely the situation in the ghost condensate model, where such modification does not lead to any problems (provided $a$ is positive), because the resulting degree of freedom has a healthy (positive) kinetic term. However, in many of other cases discussed in section 3 this is not the case and the resulting degree of

\footnotetext{
${ }^{7}$ We are thankful to Riccardo Rattazzi for numerous fruitful discussions of these stability issues and for the collaboration on the subject.
} 
freedom is a ghost. Effect of higher derivative terms on the dispersion relation (3.10) is even worse. Indeed, one obtains dispersion relation of the form

$$
p^{2}=b \frac{\omega^{4}}{\Lambda^{2}}+\cdots
$$

inevitably leading to both quantum and classical instability.

The above discussion implies that the generic effect of higher derivative terms is to make the actual cutoff of the theory much lower than the naive value $\Lambda$. It is worth stressing, that this does not imply that one cannot assume that the structure of the theory in the UV is such, that coeffcients $a, b$ and their higher order analogues are very small, so that they do not lead to any pathologies in the low-energy effective theory below cutoff $\Lambda$, due to the same reasons as small departures from fine-tuning relations at the quadratic level. Clearly, from the viewpoint of low-energy effective field theory this is a very fine-tuned situation, but there is nothing principally wrong in considering such a situation.

While, a priori, there is nothing wrong in considering a fine-tuned situation, it is worth looking for some symmetry principle ensuring the desired fine-tunings and thus making massive gravity well behaved without assuming any fine-tuning relations in the Goldstone action (besides those, following from the symmetries). An example of massive gravity having this attractive property of the UV insensitivity is a ghost condensate. This is achieved by requiring that there is a residual invariance under time-dependent spatial reparametrizations (1.1).

Besides the purely esthetic attractiveness this property of UV insensitivity may be very important from the practical viewpoint as well. Indeed, eventually, we are interested in the study of the non-trivial curved backgrounds in massive gravity, and in the absence of a symmetry principle there is no guarantee, that, say, the cosmological expansion would not drive a sysetm out of the safe region where fine-tuning relations hold.

To illustrate this, let us consider the following simple choice of function $F$ in the Goldstone action (2.10),

$$
F=P(X)+\left(\delta_{i j} Y^{i j}\right)^{2} .
$$

This is just a ghost condensate action coupled to three free scalar fields $\phi^{i}$. It is straightforward to check that fine-tuning condition $m_{0}^{2}=0$ implies that

$$
P_{X}+2 X P_{X X}=0
$$

and that for a large class of functions $P$ one can get a massive gravity in phase $m_{0}^{2}=0$ using this type of actions for Goldstones. On the other hand, equation for field $\phi^{0}$ in the expanding Universe in this model is the same as in the case of ghost condensate,

$$
\partial_{t}\left(a^{3} P_{X} \partial_{t} \phi^{0}\right)=0
$$

implying that at late stages of the expansion a system is driven either to a point

$$
\partial_{t} \phi^{0}=0
$$


or to the ghost condensate point

$$
P_{X}=0 \text {. }
$$

(in both cases $Y$ goes to zero). However, it is straightforward to check that it is not possible to move from the vicinity of the point where condition (4.7) is satisfied to points (4.9), (4.10) without crossing regions where instabilities are not suppressed by any small parameter.

So, let us try to see whether it is possible to protect fine-tuning relations obtained in section 3 by residual reparametrization symmetries. In each of the phases found there one of the conditions $m_{0}^{2}=0, m_{1}^{2}=0$ or $m_{2}^{2}=m_{3}^{2}$ holds. Let us see what symmetries can protect each of these relations.

Let us start from the relation $m_{1}^{2}=0$. It can be protected by one of the following residual reparametrization symmetries

$$
\begin{aligned}
x^{i} & \rightarrow x^{i}+\xi^{i}(t, x) \\
x^{i} & \rightarrow x^{i}+\xi^{i}(t) \\
t & \rightarrow t+\xi^{0}(t, x) \\
t & \rightarrow t+\xi^{0}(x) .
\end{aligned}
$$

First symmetry (4.11) is just a residual symmetry of the ghost condensate, so we will not discuss it here.

At the linear level the second symmetry (4.12) implies invariance under arbitrary timedependent shifts of the $\pi^{i}$ fields. In other words, field configuration

$$
\pi^{0}=0, \quad \pi^{i}=\pi^{i}(t)
$$

is a solution of linearized field equations, provided there is a residual reparametrization symmetry (4.12). In particular, this implies, that for arbitrary choice of higher derivative terms, compatible with symmetry (4.12), the dispersion relation in the vector sector is (at non-zero $m_{2}^{2}$ )

$$
p^{2}\left(1+a \frac{\omega^{2}}{\Lambda^{2}}+b \frac{p^{2}}{\Lambda^{2}}+\cdots\right)=0,
$$

where $a$ and $b$ are again some coefficients of order one. This is in agreement with what we found at the one derivative level, and implies that there are no propagating light vector degrees of freedom in this case.

Similarly, in the scalar sector symmetry (4.12) implies, that for generic higher derivative terms the on-shell condition in the scalar sector has the following form

$$
p^{2}\left(\omega^{2}+a \frac{p^{4}}{\Lambda^{2}}+\cdots\right)=0,
$$

where the lowest order term is determined by our explicit analysis in subsection 3.5 . Therefore, similarly to the case of ghost condensate, there is one propagating degree of freedom in the scalar sector with peculiar dispersion relation

$$
\omega^{2} \propto p^{4}
$$


in this case. In order this degree of freedom had a healthy sign before kinetic term the derivative of the eigenvalue of matrix (3.34) having zero at $\nu^{2}=0$ should be positive at this point. It is straightforward to check that this is the case, provided

$$
m_{0}^{2}-\frac{m_{4}^{4}}{\left(m_{3}^{2}-m_{2}^{2}\right)}>0 .
$$

The point $m_{3}^{2}=m_{2}^{2}$ is rather special — here both eigenvalues of matrix (3.34) have square root branchings at $\nu^{2}=0$. Perhaps, this case deserves further study.

Thus, insisting on the residual symmetry (4.12), one obtains a generalization of the ghost condensate theory sharing all attractive features of the latter. On the other hand this model has a number of new features. For instance, tensor gravitational waves are massive here. Clearly, this theory deserves a separate detailed study.

Symmetry (4.13) implies that all masses but $m_{2}^{2}$ and $m_{3}^{2}$ vanish. As we discussed in subsection 3.5 in this case matrix $M$ is degenerate, so that Goldstone sector is not well behaved in the decoupling limit.

Finally, symmetry (4.14) does not allow to put any restrictions on higher-derivative corrections to the dispersion relation in the vector sector, so it does not allow to avoid fine-tuning. Similarly, in the scalar sector it implies that dispersion relation has a form

$$
\omega^{2}\left(p^{2}+a \frac{\omega^{4}}{\Lambda^{2}}+\cdots\right)=0
$$

that does not protect from the instabilities.

Let us now discuss symmetries protecting fine-tuning relation $m_{2}^{2}=m_{3}^{2}$. Besides symmetry (4.11), leading to ghost condensate, this fine-tuning relation can be protected by a less restrictive residual symmetry

$$
x^{i} \rightarrow x^{i}+\xi^{i}(x)
$$

Actually, this symmetry implies a stronger condition

$$
m_{2}^{2}=m_{3}^{2}=m_{4}^{2}=0 .
$$

In the vector sector it protects from any instabilities in the low-energy effective theory, leading to the dispersion relation

$$
\omega^{2}\left(1+a \frac{\omega^{2}}{\Lambda^{4}}+\cdots\right)
$$

provided $m_{1}^{2} \neq 0$. In the scalar sector symmetry (4.19) implies the following form of the dispersion relation (at the lowest order we are using the result of subsection 3.3.

$$
\omega^{2}\left(\omega^{2}+a \frac{p^{4}}{\Lambda^{2}}+\cdots\right)=0 .
$$

Consequently, we again obtain one propagating degree of freedom in the scalar sector with dispersion relation (4.17). To understand when this mode has a healthy kinetic term let 

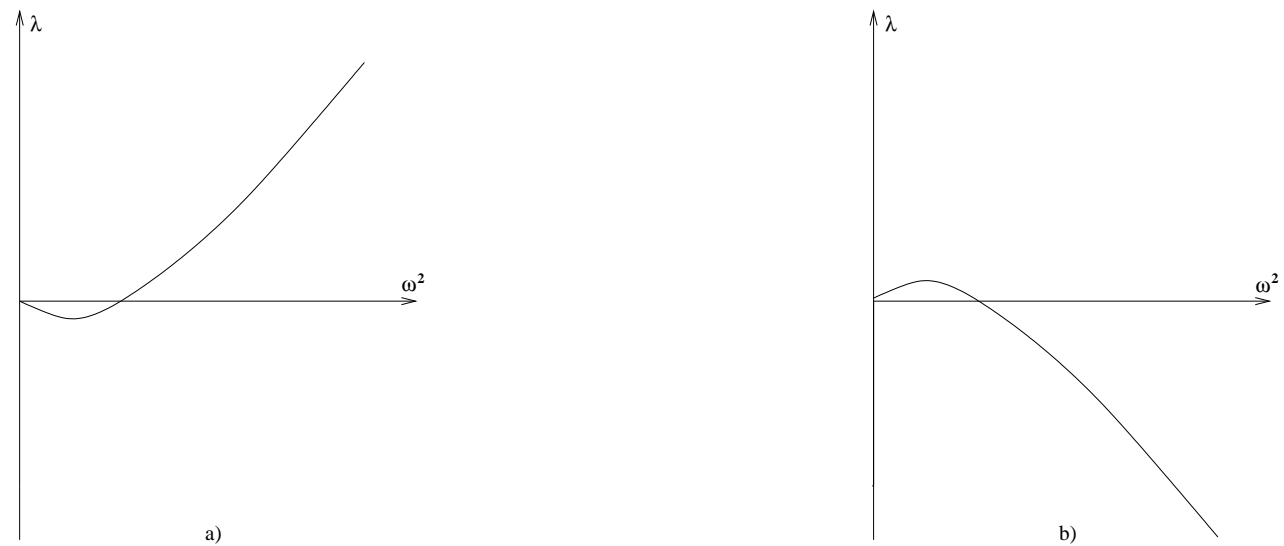

Figure 1: Splitting of zeroes of the eigenvalue of the matrix $M$ due to higher-derivative terms in the presence of residual symmetry (4.19) in two different cases: $(a) m_{1}^{2}, m_{0}^{2}>0,(b) m_{1}^{2}, m_{0}^{2}<0$.

us look at the behavior of the eigenvalues of matrix (3.8) when masses $m_{2}^{2}, m_{3}^{2}$ and $m_{4}^{2}$ are zero. At this point one of the eigenvalues has a double zero at $\omega^{2}=0$ while another has no zeros at all. Higher order terms in the dispersion relation (4.22) lead to the splitting of the double zero into two simple zeroes as shown in figure 1 (for negative value of the coefficient $a$ in eq. (4.22)). Now, for positive $m_{1}^{2}$ the propagating mode has a healthy kinetic term in the situation shown in the left panel of figure 1 while for negative $m_{1}^{2}$ in the situation shown in the right panel of figure 15. Straightforward calculation shows that propagating mode is not a ghost provided

$$
m_{0}^{2} m_{1}^{2}>0 .
$$

This UV insensitive theory resembles ghost condensate even more, because tensor mode is massless in both cases. Also, in both cases graviton mass has a form of a gauge fixing term and thus higher order terms (non-linear, or higher derivative) are needed to see modification of gravity.

Finally, let us discuss whether it is possible to make use of a residual gauge symmetry to avoid fine-tunings in the phase with $m_{0}^{2}=0$. Besides the symmetry (4.13) leading to the degeneracy in the Goldstone sector one may try the following symmetry

$$
t \rightarrow t+\xi^{0}(t)
$$

This symmetry implies that

$$
m_{0}^{2}=m_{4}^{2}=0 .
$$

Unfortunately, in the scalar sector this symmetry implies the dispersion relation of the form

$$
p^{2}\left(p^{2}+a \frac{\omega^{4}}{\Lambda^{2}}+\cdots\right)=0
$$

and thus is not enough to obtain a UV insensitive theory.

To summarize, we found two new examples of the UV insensitive massive gravities. This is the whole phase $m_{1}^{2}=0$ and a part of the boundary of $m_{2}^{2}=m_{3}^{2}$ phase, where 
condition (4.20) holds. In both cases the residual symmetry group is a subgroup of the residual symmetry of the ghost condensate. Both theories deserve further detailed study.

Clearly, our analysis does not exhaust all possible subgroups of the diffeomorphism group. It is worth studying whether other interesting possibilities exist.

\section{Mixing with gravity and absence of the $\mathrm{vDVZ}$ discontinuity}

Let us discuss now, how mixing with gravity may affect the above conclusions and show that the vDVZ discontinuity is absent if graviton masses are in the ranges found in section 3 .

Let us start from the discussion of the vDVZ discontinuity. The complete linearized field equations in massive gravity can be presented schematically as follows

$$
\left(\mathcal{M}_{0}+\mathcal{M}_{m}\right)\left(\begin{array}{c}
h_{\mu \nu} \\
\pi_{\mu}
\end{array}\right)=\left(\begin{array}{c}
T_{\mu \nu} \\
0
\end{array}\right)
$$

where $T_{\mu \nu}$ is the energy-momentum tensor of matter, we assumed that there are no sources for the Goldstone fields $\pi_{\mu}$; matrix $\mathcal{M}_{0}$ is diagonal

$$
\mathcal{M}_{0}=\left(\begin{array}{cc}
M_{P l}^{2} \mathcal{D}_{E}^{2} & 0 \\
0 & \Lambda^{4} \mathcal{D}_{G}^{2}
\end{array}\right),
$$

and matrix $\mathcal{M}_{m}$ accounts for graviton masses and kinetic mixings between Goldstones and gravitons,

$$
\mathcal{M}_{m}=\left(\begin{array}{cc}
\Lambda^{4} \mathcal{O} & \Lambda^{4} \mathcal{D}_{m} \\
\Lambda^{4} \mathcal{D}_{m} & 0
\end{array}\right) .
$$

Here we assumed that gauge symmetry is fixed in the graviton sector (for instance, using harmonic gauge), so that $\mathcal{D}_{E}^{2}$ is the ordinary two-derivative graviton kinetic operator following from the Einstein-Hilbert action with gauge fixing terms, $\mathcal{D}_{G}^{2}$ is the two derivative kinetic operator in the Goldstone sector, $\mathcal{O}$ is a dimensionless matrix, determining the tensor structure of the graviton mass matrix, and $\mathcal{D}_{m}$ is a one-derivative operator describing mixing between Goldstones and graviton. For graviton mass parameters found in section 3 both operators $\mathcal{D}_{E}^{2}$ and $\mathcal{D}_{G}^{2}$ are invertible at general values of energy $\omega$ and momentum $p$. Therefore, one can present matrix $\mathcal{M}$ in the following form

$$
\mathcal{M}=\mathcal{M}_{0}^{1 / 2}\left(\mathbf{1}+\left(\begin{array}{cc}
\frac{\Lambda^{4}}{M_{P l}^{2}} \mathcal{D}_{E}^{-1} \mathcal{O} \mathcal{D}_{E}^{-1} & \frac{\Lambda^{2}}{M_{P l}} \mathcal{D}_{E}^{-1} \mathcal{D}_{m} \mathcal{D}_{G}^{-1} \\
\frac{\Lambda^{2}}{M_{P l}} \mathcal{D}_{G}^{-1} \mathcal{D}_{m} \mathcal{D}_{E}^{-1} & 0
\end{array}\right)\right) \mathcal{M}_{0}^{1 / 2}
$$

This presentation makes it manifest, that, far from the on-shell values of energy and momentum found in section 3 , the metric induced by the source $T_{\mu \nu}$ differs from that in the pure Einstein theory by small corrections, vanishing when the scale of graviton masses

$$
m=\frac{\Lambda^{2}}{M_{P l}}
$$

goes to zero. In particular, this is true in the newtonian regime, provided energy $\omega$ is much larger than the graviton mass scale

$$
m \ll \omega \ll p<\Lambda .
$$


Hence, on time and length scales shorter than the inverse graviton mass one reproduces the usual newtonian limit of gravity and vDVZ discontinuity is absent.

Let us check now whether mixing with gravity can introduce dangerous instabilities for the "safe" phases found in section 3. The most straightforward way to do this is, following ref. [15], to study the spectrum of massive gravity in the unitary gauge in each of the cases. Namely, one writes

$$
\begin{aligned}
h_{00} & =\psi \\
h_{0 i} & =u_{i}+\partial_{i} v \\
h_{i j} & =\chi_{i j}+\left(\partial_{i} s_{j}+\partial_{j} s_{i}\right)+\partial_{i} \partial_{j} \sigma+\delta_{i j} \tau
\end{aligned}
$$

where $\xi_{i j}$ is a transverse-traceless tensor, $u_{i}$ and $v_{i}$ are transverse vectors and other fields are three-dimensional scalars. Let us start from the vector sector. Here one has the following action 15

$$
L_{v}=M_{P l}^{2}\left(s_{i} \partial_{0}^{2} \partial_{j}^{2} s_{i}-u_{i} \partial_{j}^{2} u_{i}+2 u_{i} \partial_{j}^{2} \partial_{0} s_{i}+m_{1}^{2} u_{i} u_{i}+m_{2}^{2} s_{i} \partial_{j}^{2} s_{i}\right) .
$$

Dispersion relation following from this action is

$$
m_{1}^{2} p^{2} \omega^{2}-m_{2}^{2} p^{2}\left(p^{2}+m_{1}^{2}\right)=0 .
$$

Consequently, the only effect of mixing with gravity in the vector sector is the emergence of the mass gap $m_{2}^{2}$.

Let us now consider the scalar sector. Here, at general values of masses, one has the following quadratic action in the unitary gauge

$$
\begin{aligned}
L=\frac{M_{P l}^{2}}{2} & \left(4\left(\psi-2 \partial_{0} v+\partial_{0}^{2} \sigma\right) \partial_{i}^{2} \tau+6 \tau \partial_{0}^{2} \tau-2 \tau \partial_{i}^{2} \tau+m_{0}^{2} \psi^{2}+2 m_{1}^{2}\left(\partial_{i} v\right)^{2}-\right. \\
& \left.-m_{2}^{2}\left(\left(\partial_{i}^{2} \sigma\right)^{2}+2 \tau \partial_{i}^{2} \sigma+3 \tau^{2}\right)+m_{3}^{2}\left(\partial_{i}^{2} \sigma+3 \tau\right)^{2}-2 m_{4}^{2} \psi\left(\partial_{i}^{2} \sigma+3 \tau\right)\right) .
\end{aligned}
$$

Let us start our analysis without assuming any fine-tuning relations between masses. Fields $\psi$ and $v$ enter action (5.9) without time derivatives [15] (the term propartional to $\partial_{0} v \partial_{i}^{2} \tau$ in eq. (5.9) may be written as $\left.v \partial_{0} \partial_{i}^{2} \tau\right)$. Field equations resulting from variation with respect to these fields can be written as

$$
\begin{aligned}
v & =\frac{2}{m_{1}^{2}} \partial_{0} \tau \\
\sigma & =\frac{2}{m_{4}^{2}} \tau-\frac{3}{\partial_{i}^{2}} \tau+\frac{m_{0}^{2}}{m_{4}^{2}} \frac{1}{\partial_{i}^{2}} \psi .
\end{aligned}
$$

Plugging expressions (5.10) and (5.11) back into action we arrive at the following lagrangian for the two remaining fields $\tau$ and $\psi$ (we will discuss later what happens if $m_{1}^{2}=0$ or $\left.m_{4}^{2}=0\right)$,

$$
L=\frac{M_{P l}^{2}}{2}\left(\left(\frac{8}{m_{4}^{2}}-\frac{8}{m_{1}^{2}}\right) \partial_{i}^{2} \tau \partial_{0}^{2} \tau-4 \frac{m_{2}^{2}-m_{3}^{2}}{m_{4}^{4}}\left(\partial_{i}^{2} \tau\right)^{2}-6 \tau \partial_{0}^{2} \tau+\left(8 \frac{m_{2}^{2}}{m_{4}^{2}}-2\right) \tau \partial_{i}^{2} \tau-\right.
$$




$$
\begin{aligned}
& -6 m_{2}^{2} \tau^{2}+4 \frac{m_{0}^{2}}{m_{4}^{2}} \tau \partial_{0}^{2} \psi-4 \frac{m_{0}^{2}}{m_{4}^{4}}\left(m_{2}^{2}-m_{3}^{2}\right) \tau \partial_{i}^{2} \psi- \\
& \left.-\left(m_{0}^{2}+\frac{m_{0}^{4}}{m_{4}^{4}}\left(m_{2}^{2}-m_{3}^{2}\right)\right) \psi^{2}+4 m_{0}^{2} \frac{m_{2}^{2}}{m_{4}^{2}} \tau \psi\right) .
\end{aligned}
$$

Let us first consider the phase $m_{0}^{2}=0$, when all terms in the second line of eq. (2.12) vanish. But before actually setting them to zero, the following comment is in order. Straightforward inspection of action (5.12) shows, that the dispersion relation of the mode which is nondynamical at $m_{0}^{2}=0$ takes the following form at small (much smaller than all other masses) non-vanishing $m_{0}^{2}$,

$$
m_{0}^{2} \omega^{2}=-\frac{3}{2} \frac{m_{4}^{4}}{\mu_{0}^{2}}\left(p^{2}+\mu_{0}^{2}\right)
$$

where parameter $\mu_{0}^{2}$ is defined as follows

$$
\frac{4}{m_{4}^{2}}-\frac{4}{m_{1}^{2}}=\frac{3}{\mu_{0}^{2}}
$$

We see, that mixing with gravity automatically provides an IR regulator of the type (1.3) needed to stabilize the Goldstone sector upon small departure from the surface $m_{0}^{2}=0$.

Stability of the action (5.12) in the bulk of the $m_{0}^{2}=0$ phase was studied in ref. [15]. As we mentioned in subsection 3.2 the only difference of the results of this analysis with ours is that extra stability condition

$$
4 m_{2}^{2}>m_{4}^{2}
$$

was imposed in that work. If this condition is violated, the term proportinal to $\tau \partial_{i}^{2} \tau$ enters action (5.12) with negative sign. Depending on other parameters, this may lead to the Jeans-like instability in the finite region of small momenta and frequencies, $\omega, p \sim$ $m$. Presence of such instability does not invalidate the use of the low-energy effective theory with cutoff $\Lambda$ for time scales shorter than $\sim m^{-1}$ and may even lead to interesting phenomenological consequences. So we do not think that one should a priori disregard mass values violating condition (5.14).

Let us see now what are the effects of mixing with gravity at the boundaries of the phase $m_{0}^{2}=0$. If $m_{1}^{2}=m_{4}^{2}$, then action (5.12) describes a propagating degree of freedom with the following dispersion relation (cf. ref. [15])

$$
\omega^{2}=\left(p^{2}+z \mu_{1}^{2}\right) \frac{p^{2}}{\mu_{1}^{2}}+m_{2}^{2}
$$

where

$$
2 \frac{m_{2}^{2}-m_{3}^{2}}{m_{4}^{4}}=\frac{3}{\mu_{1}^{2}}, \quad 4 \frac{m_{2}^{2}}{m_{4}^{2}}-1=3 z .
$$

We see, that in this case mixing with gravity invalidates our conclusion that there are no propagating degrees of freedom in the scalar sector. The new degree of freedom has a healthy kinetic term and does not lead to the vDVZ discontinuity provided $m_{2}^{2} \neq m_{3}^{2}$. In order to avoid rapid classical instabilities, one needs, however,

$$
\mu_{1}^{2}>0,
$$


or, equivalently,

$$
m_{2}^{2}>m_{3}^{2}
$$

This condition was impossible to obtain from inspecting the Goldstone lagrangian in the decoupling limit at $m_{1}^{2}=m_{4}^{2}$.

If $m_{2}^{2}=m_{3}^{2}$ the dispersion relation obtained in ref. [15] reduces to

$$
\omega^{2}=\mu_{0}^{2} \frac{z p^{2}+m_{2}^{2}}{p^{2}+\mu_{0}^{2}}
$$

Again we see that mixing with gravity results in the emergence of a new propagating degree of freedom. This degree of freedom does not lead to any rapid instabilities, provided

$$
\mu_{0}^{2}>0
$$

or, equivalently,

$$
m_{1}^{2}>m_{4}^{2}>0 .
$$

As before, this condition is impossible to deduce from the Goldstone lagrangian alone. Finally, if in addition to $m_{0}^{2}=0$ one has $m_{4}^{2}=0$, then eq. (5.11) implies that $\tau=0$. Then action in the scalar sector takes the following form

$$
L=\frac{M_{P l}^{2}}{2}\left(m_{3}^{2}-m_{2}^{2}\right)\left(\partial_{i}^{2} \sigma\right)^{2}
$$

and does not describe any propagating degrees of freedom. Consequently, mixing with gravity does not bring nothing new in this case.

Let us now discuss effects of mixing with gravity for phase $m_{1}^{2}=0$, protected by the residual gauge symmetry (4.11). Here eq. (5.10) implies, that either $\omega=0$, or $\tau=0$. In the latter case, one uses eq. (5.11) to eliminate $\psi$ and arrives at the following action for $\sigma$,

$$
L=\frac{M_{P l}^{2}}{2}\left(m_{3}^{2}-m_{2}^{2}-\frac{m_{4}^{4}}{m_{0}^{2}}\right)\left(\partial_{i}^{2} \sigma\right)^{2}
$$

which does not describe any propagating fields. So in this case mixing with gravity does not introduce any extra degrees of freedom.

We believe, that the above discussion is representative enough to demonstrate what are the possible effects of mixing with gravity. So we leave the detailed study of the consequences of this mixing in the $m_{2}^{2}=m_{3}^{2}$ phase and for $m_{2}^{2}=0$ with negative $m_{1}^{2}$ beyond the scope of the current paper. Instead, we just consider the point corresponding to the second UV insensitive theory found in section 6 .

Before doing that, let us mention one more possible effect of mixing, that was not realized in the phase $m_{0}^{2}=0$. Namely, it follows from eq. (5.12) that in the phase with $m_{2}^{2}=m_{3}^{2}$ (and non-zero $m_{0}^{2}$ ) a generic consequence of mixing with gravity is that sixth polarization mode of the graviton becomes dynamical. Discussion in subsection 3.3 suggests that this mode may be a ghost or lead to classical instabilities. Presumably, there are regions of mass parameters where this is not dangerous. Our discussion in section 1 
implies, that what one needs, is that all quanta of this mode are very soft (cf. dispersion relation (5.18) ) and, consequently, the corresponding instability rate is very slow. ${ }^{8}$

For point $m_{2}^{2}=m_{3}^{2}=m_{4}^{2}=0$, protected by the symmetry (4.19), one uses eq. (5.11) to express $\psi$ through $\tau$ and eq. (5.10) to express $v$ through $\tau$. As a result one arrives at the following action

$$
L=\frac{M_{P l}^{2}}{2}\left(-\frac{4}{m_{0}^{2}}\left(\partial_{i}^{2} \tau\right)^{2}-\frac{8}{m_{1}^{2}}\left(\partial_{0} \partial_{i} \tau\right)^{2}+4 \partial_{0} \partial_{i} \tau \partial_{0} \partial_{i} \sigma+6\left(\partial_{0} \tau\right)^{2}+2\left(\partial_{i} \tau\right)^{2}\right) .
$$

Variation of this action with respect to $\sigma$ implies that $\tau=0$ if both $\omega$ and $p$ are non-zero. Then variation with respect to $\tau$ gives $\sigma=0$. So mixing with gravity does not introduce new propagating modes in this case as well.

\section{Discussion}

In this final section let us first briefly discuss what kind of cosmology can one expect in models of massive gravity discussed above. A hope that long scale modification of gravity may explain the observed acceleration of the cosmological expansion rate, or, at least, relate it to effects testable at shorter scales (such as the anomalous precession of the Moon [20, 21]) was the main driving force for the interest to massive gravity and its brane world analogues during the last few years. The common lore, supported by the brane world models (e.g., by the DGP model [10]) is that modification of the gravitational potential at the distance scale $r_{c}$ is related to the modification of the Friedmann equation at the value of the Hubble expansion rate $H$ of order $r_{c}^{-1}$.

An example of the ghost condensate suggests that in four-dimensional models of massive gravity situation may be completely different. Indeed, at the linear level this model is characterized by distance scale $r_{c}$ and time scale $t_{c}$, such that gravitational potential of static sources is strongly modified at distance and time scales larger than $r_{c}$ and $t_{c}$ correspondingly. On the other hand, the homogeneous Friedmann equation in this model is completely equivalent to the standard one (modulo, possibly, the presence of additonal dust-like component, with relative density depending on the initial conditions for the scalar field) for arbutrary value of the Hubble rate.

To illustrate that this is not a peculiar property of the ghost condensate, let us consider Lorentz-violating gravity with function $F$ (see eq. (2.10) of the following form

$$
F=\Lambda^{4} F\left(X Y^{3}\right) \text {. }
$$

In the unitary gauge this means, that we are adding to the Einstein action the following non-covariant term

$$
\int d^{4} x \Lambda^{4} \sqrt{-g} F\left(g^{00}\left(\operatorname{Tr} g^{i j}\right)^{3}\right) .
$$

We will comment later what was the reason to assume this particular form of the action. At the moment, let us just note that independence of function $F$ on $Z$ can be ensured, e.g.,

\footnotetext{
${ }^{8}$ Note, that this may imply additional limitations on the possible values of masses, that are not seen in the decoupling limit.
} 
by time inversion symmetry. Also, for the solutions we discuss here $V^{i}=0$, so possible dependence of $F$ on $V^{i}$ would not affect our discussion. Finally, there is a global symmetry

$$
\phi^{0} \rightarrow \lambda \phi^{0}, \quad \phi^{i} \rightarrow \lambda^{-1 / 3} \phi^{i}
$$

protecting dependence of function $F$ on the combination $X Y^{3}$ at the quantum level.

Flat homogeneous cosmologies in massive gravity are described by the following ansatz for the metric and Goldstones,

$$
\begin{aligned}
d s^{2} & =d t^{2}-a^{2}(t) d x^{i} d x^{i} \\
\phi^{0} & =\phi^{0}(t) \\
\phi^{i} & =\Lambda^{2} x^{i}
\end{aligned}
$$

In particular, $Y=1 / a^{2}$ and goes to zero as the Universe expands, so one may suspect that effect of all masses but $m_{0}^{2}$ is negligible at late times. We will see, however, that this is not necessarily the case. Ansatz (6.2) automatically satisfies $\phi^{i}$ field equations, while $\phi^{0}$ equation reads as follows

$$
\partial_{t}\left(a^{3} F^{\prime}\left(X Y^{3}\right) Y^{3} \partial_{t} \phi^{0}\right)=\partial_{t}\left(F^{\prime}\left(X a^{-6}\right) X^{1 / 2} a^{-3}\right)=0 .
$$

A solution to this equation is

$$
X=x_{0} a^{6}
$$

or

$$
\phi_{0}(t)=x_{0}^{1 / 2} \int^{t} d t a^{3}(t) .
$$

Combination $X Y^{3}$ entering the Goldstone action remains constant for this solution. On the first hand this implies that graviton masses are constant during the cosmological evolution. In particular, if some fine-tuning relation were true in the begining of expansion it keeps to be true during the whole history of the Universe. For instance, it is straightforward to check, that action (6.1) allows cosmological solutions in the $m_{0}^{2}=0$ phase of massive gravity.

On the other hand, time-independence of $X Y^{3}$ implies that contribution of the Goldstone sector to the energy-momentum tensor is also constant. In other words, this contribution is either zero or cosmological constant. So this is an example of the model where all graviton modes are massive, but flat homogeneous solutions are the same as in the Einstein gravity. Should one be disappointed by this fact? We believe that the answer is not. This just implies that the value of the graviton mass may be quite large, affecting growth of primordial perturbations, structure formation, or even gravitational dynamics at the (super)galactic scales.

Even more intriguingly, this opens up a possibility that the effects of backreaction of small scale inhomgeneities on the expansion rate of the Universe can be quite substantial in massive gravity. Actually, the backreaction effects in conventional Einstein theory are not completely understood, but it appeares that the likely conclusion is that they are very small in the realistic Universe (see, e.g. refs. [22]-[25] for a recent discussion). However, this may 
be completely different in massive gravity. Indeed, in this case there is at least one extra length scale in the problem - inverse mass of the graviton. Now imagine the Universe consisting of the heavy point-like galaxies at large distances from each other. Then, when the distances between neighboring galaxies are larger than the inverse graviton mass, the pairwise interactions between galaxies are practically negligible (at least, in the $m_{0}^{2}=0$ phase, where the gravitational potential is of the Yukawa type [15]). Is it possible to use in this case the homogeneous approximation in cosmology? The answer to this question is not clear to us.

An indirect hint that there may be a subtlety here is coming from the attempt to find closed or open homogeneous cosmological solutions in the model (b.1). In this case instead of a distance between galaxies there is another extra length scale in the problem - curvature radius. ${ }^{9}$ It is straightforward to see, however, that the corresponding ansatz does not satisfy all the equations (except the case of the de Sitter space, when there are extra symmetries). This implies that closed or open Universe is necessarily inhomogeneous on the scale of its curvature radius in massive gravity with action (6.1).

To conclude this brief discussion of the cosmology in massive gravity, it is worth noting that time-independence of the graviton mass during the cosmological expansion is by no means a general property of massive gravity. Actually, the particular form of the action (6.1) was derived from the requirement that graviton mass is constant. The study of cosmological consequences of the time-dependent graviton mass may provide new surprises.

Finally, let us stress that there is a large number of problems not addressed in the current paper. First of all, there are still a lot of questions about stability of massive gravity. For instance, our analysis of mixing with gravity in section 0 covers only the phases $m_{0}^{2}=0$ (where it only slightly extends that performed in ref. [15]), $m_{1}^{2}=0$ and a point with enhanced symmetry in the phase with $m_{2}^{2}=m_{3}^{2}$. More detailed analysis of the phases with $m_{2}^{2}=m_{3}^{2}$ and $m_{2}^{2}=0$ with negative $m_{1}^{2}$ is needed to decide which regions of parameters identified in section 3 actually correspond to tractable low-energy effective theories with cutoff $\Lambda$. Also, it is necessary to include higher derivative terms in this analysis. A particulary interesting question is whether two new UV insensitive theories found here may avoid Jeans-like instability present in the ghost condensate case. Furthermore, the analysis of stability of non-linear solutions (on the first hand, cosmological ones and massive analogues of the Schwartzchild metric) is needed to conclusively decide on the phenomenological acceptability of models discussed here.

Also, in the current paper we concentrated on the issues of consistency and stability and leave aside the analysis of phenomenology of modification of gravity in models studied here. Definitely, one of the most pressing questions is what are the actual limits on the graviton mass parameters. It seems likely, that to obtain an accurate answer to this question a detailed study of growth of primordial perturbations and structure formation in massive gravity is needed.

Another circle of questions is what are the limits on the direct couplings between Goldstone sector and Standard Model fields and what are the implications of massive

\footnotetext{
${ }^{9}$ We thank Dominik Schwarz for suggesting us to look for open or closed solutions.
} 
gravity for inflation (see, refs. [26, 27 where these issues were studied in the case of ghost condensate). We hope to address these and other related questions in future.

\section{Acknowledgments}

We appreciate stimulating discussions and correspondence with Peter Tinyakov, Igor Tkachev, Misha Shaposhnikov, Dominik Schwarz and Valery Rubakov. Special thanks are to Riccardo Rattazzi for numerous fruitful discussions and collaboration on some of the subjects discussed here. We thank organizers of the 9th Summer Institute at the Gran Sasso Laboratory (in particular, Zurab Berezhiani), where part of this work was done, for a kind hospitality.

\section{References}

[1] N. Arkani-Hamed, H. Georgi and M.D. Schwartz, Effective field theory for massive gravitons and gravity in theory space, Ann. Phys. (NY) 305 (2003) 96 hep-th/0210184.

[2] H. van Dam and M.J.G. Veltman, Massive and massless Yang-Mills and gravitational fields, Nucl. Phys. B 22 (1970) 397.

[3] V.I. Zakharov, Linearized gravitation theory and the graviton mass, Sov. Phys. JETP Lett. $12(1970) 312$.

[4] A.I. Vainshtein, To the problem of nonvanishing gravitation mass, Phys. Lett. B 39 (1972) 393.

[5] D.G. Boulware and S. Deser, Can gravitation have a finite range?, Phys. Rev. D 6 (1972) 3368 .

[6] A. Nicolis and R. Rattazzi, Classical and quantum consistency of the DGP model, J. High Energy Phys. 06 (2004) 059 hep-th/0404159.

[7] G. Dvali, Infrared modification of gravity, hep-th/0402130.

[8] M.A. Luty, M. Porrati and R. Rattazzi, Strong interactions and stability in the DGP model, J. High Energy Phys. 09 (2003) 029 hep-th/0303116.

[9] V.A. Rubakov, Strong coupling in brane-induced gravity in five dimensions, hep-th/0303125.

[10] G.R. Dvali, G. Gabadadze and M. Porrati, $4 D$ gravity on a brane in 5D Minkowski space, Phys. Lett. B 485 (2000) 208 hep-th/0005016.

[11] N. Arkani-Hamed, H.-C. Cheng, M.A. Luty and S. Mukohyama, Ghost condensation and a consistent infrared modification of gravity, J. High Energy Phys. 05 (2004) 074 hep-th/0312099.

[12] S.L. Dubovsky, Star tracks in the ghost condensate, JCAP 07 (2004) 009 hep-ph/0403308.

[13] M. Peloso and L. Sorbo, Moving sources in a ghost condensate, Phys. Lett. B 593 (2004) 25 hep-th/0404005.

[14] A.V. Frolov, Accretion of ghost condensate by black holes, hep-th/0404216.

[15] V. Rubakov, Lorentz-violating graviton masses: getting around ghosts, low strong coupling scale and VDVZ discontinuity, hep-th/0407104. 
[16] T. Damour, I.I. Kogan and A. Papazoglou, Spherically symmetric spacetimes in massive gravity, Phys. Rev. D 67 (2003) 064009 hep-th/0212155.

[17] B.M. Gripaios, Modified gravity via spontaneous symmetry breaking, J. High Energy Phys. 10 (2004) 069 hep-th/0408127.

[18] J.M. Cline, S.-y. Jeon and G.D. Moore, The phantom menaced: constraints on low-energy effective ghosts, Phys. Rev. D 70 (2004) 043543 hep-ph/0311312.

[19] B. Holdom, Accelerated expansion and the Goldstone ghost, J. High Energy Phys. 07 (2004) 063 hep-th/0404109.

[20] A. Lue and G. Starkman, Gravitational leakage into extra dimensions: probing dark energy using local gravity, Phys. Rev. D 67 (2003) 064002 astro-ph/0212083.

[21] G. Dvali, A. Gruzinov and M. Zaldarriaga, The accelerated universe and the moon, Phys. Rev. D 68 (2003) 024012 hep-ph/0212069.

[22] T. Futamase, Averaging of a locally inhomogeneous realistic universe, Phys. Rev. D 53 (1996) 681.

[23] T. Buchert, On average properties of inhomogeneous fluids in general relativity: perfect fluid cosmologies, Gen. Rel. Grav. 33 (2001) 1381 gr-qc/0102049.

[24] S. Rasanen, Dark energy from backreaction, JCAP 02 (2004) 003 astro-ph/0311257.

[25] E.W. Kolb, S. Matarrese, A. Notari and A. Riotto, The effect of inhomogeneities on the expansion rate of the universe, hep-ph/0409038.

[26] N. Arkani-Hamed, H.-C. Cheng, M. Luty and J. Thaler, Universal dynamics of spontaneous Lorentz violation and a new spin-dependent inverse-square law force, hep-ph/0407034.

[27] N. Arkani-Hamed, P. Creminelli, S. Mukohyama and M. Zaldarriaga, Ghost inflation, JCAP 04 (2004) 001 hep-th/0312100. 